\title{
Bilan de sept années de prophylaxie de la dermatophilose dans un troupeau de zébus Brahman
}

\author{
par J. BLANCOU (*)
}

\begin{abstract}
RÉSUMÉ
Les résultats des prophylaxies sanitaire et médicale sont comparés. Cette dernière semble la plus efficace, que ce soit sous forme d'une chimioprophylaxie mensuelle (par voie externe ou parentérale) ou d'une vaccination annuelle. Mais elle ne suffit pas à protéger la totalité d'un troupeau contre la maladie en élevage extensif.
\end{abstract}

Depuis la découverte par VAN SACEGHEM, en 1915, de la bactérie associée aux dermatoses des bovins congolais, les essais de traitements et de prophylaxie de l'infection par Dermatophilus congolensis se sont multipliés.

Cette abondance de travaux démontre d'elle même la complexité d'un problème encore loin d'être résolu de façon satisfaisante, ainsi que le rappelle P. PERREAU (9).

Nous nous y sommes trouvés confrontés durant 7 années consécutives (1969 à 1974), ayant en charge la prophylaxie de la maladie dans un effectif de plus de 500 zébus Brahmans ou croisés vivant en élevage extensif dans l'Ouest de Madagascar (**).

Nous leur avons appliqué la plupart des méthodes recommandées en matière de prophylaxie de la dermatophilose, et en avons essayé de nouvelles. $\mathrm{Ce}$ sont les résultats de ces essais que nous rapportons ici.

(*) Adresse actuelle : Laboratoire National de l'Elevage et de Recherches Vétérinaires, B. P. 2057, DakarHann, Rép. du Sénégal.

(**) Stations de Recherches Zootechniques de Kianjasoa et Miadana, et Ranches de la Ferme d'Etat «Omby ».

\section{ESSAIS DE PROPHYLAXIE SANITAIRE}

Suivant les recommandations de nombreux auteurs, en particulier celles de I. MACADAM (8), nous avons tenté de limiter l'extension de l'enzootie dermatophilosique en isolant et en abattant les malades.

Les résultats ont été décevants, comme le démontre le tableau I (p. 212).

Sur les stations de Kianjasoa et Miadana, l'abattage de tous les malades en fin de saison sèche a été décidé en 1969 et 1970 . On constate, d'après le tableau $I$, que cela n'a nullement réduit la morbidité pendant les années suivantes.

Sur les ranches de la Ferme d'Etat "Omby" » lisolement systématique des malades a été pratiqué à partir de 1973. Cet isolement consistait en la ségrégation de trois troupeaux distincts :

- sujets à lésions cutanées avancées;

— sujets à lésions cutanées débutantes, ou en voie de guérison;

- sujets sans lésions cutanées apparentes.

Les permutations nécessaires entre ces trois troupeaux étaient effectuées régulièrement. On peut constater, d'après les chiffres du tableau I, que ces mesures n'ont pas encore réduit le taux de morbidité. 
TABL. $\mathrm{N}^{\circ}$ I-Taux de morbidıté annuel avant ou après les mesures de prophylaxie sanitaire.

\begin{tabular}{|c|c|c|c|c|c|}
\hline \multicolumn{3}{|c|}{ Stations de Kianjasoa et de Miadana } & \multicolumn{3}{|c|}{ Ranches de la Ferme d"Etat "Orby" } \\
\hline Annëe & Prophylaxie sanutaire & Morbidité & Arnnĕe & Prophylaxie sanitaire & Morbidité \\
\hline 1968 & Néant & $17 \mathrm{p} \cdot 100$ & \multirow{2}{*}{1972} & \multirow{2}{*}{ Kêant } & \multirow{2}{*}{$45 \mathrm{p} \cdot 100$} \\
\hline 1969 & Abattage & 25 p. 100 & & & \\
\hline 1970 & Abattage & 20 p. 100 & \multirow{2}{*}{1973} & \multirow{2}{*}{ Isolement } & \multirow{2}{*}{$42 \mathrm{p} \cdot 100$} \\
\hline 1971 & Néant & $21 \mathrm{p} \cdot 100$ & & & \\
\hline 1972 & Nëant & $5 \mathrm{p} \cdot 100$ & \multirow{2}{*}{1974} & \multirow{2}{*}{ Isolement } & \multirow{2}{*}{$40 \mathrm{p} \cdot 100$} \\
\hline 1973 & Néant & $5 \mathrm{p} .100$ & & & \\
\hline
\end{tabular}

L'échec de cette méthode, qui reste assez contraignante, est même parfois spectaculaire. C'est ainsi que sur 45 taureaux d'un troupeau "sain " de la Ferme d'État «Omby », isolé en octobre, 36 étaient atteints de formes aiguës de dermatophilose en novembre. Par ailleurs le regroupement des malades en un seul troupeau, à moins que chaque sujet ne puisse être isolé individuellement, entraîne le plus souvent une aggravation collective de leur cas, et accroît les mortalités $(*)$.

Ces observations confirment le rôle mineur de la contagion directe en matière de dermatophilose. Cette contagion se ferait-elle par contact avec des porteurs sains, comme le suggère GRABER (6), ou indirectement par des bactéries d'apport extérieur? Nous penchons plutôt pour la seconde hypothèse puisque des Brahmans parfaitement sains, importés des ÉtatsUnis, et sans aucun contact direct avec des bovins indigènes, ont contracté la maladie 14 mois après leur arrivée dans les ranches.

Par ailleurs, l'action des oiseaux piqueboufs (Buphagus africanus) ou des arbres épineux est nulle à Madagascar, ce qui est du reste une démonstration de leur faible importance dans l'épizootiologie de la dermatophilose.

L'efficacité de la prophylaxie sanitaire semble, pour toutes ces raisons, très réduite à Madagascar, du moins en matière d'élevage extensif. Ce n'est qu'en élevage intensif que l'on pourrait parvenir à isoler individuellement les animaux et les protéger contre les intempéries et le contact éventuel de matières infectées.

(*) L'expérience nous a prouvé que lorsque plus de la moitié d'un effectif bovin est atteint, il est pratiquement très difficile d'assainir le troupeau. ll faut éviter à tout prix d'atteindre ce seuil critique.
Sans doute, seul l'abattage de tous les sujets potentiellement réceptifs à la maladie constituerait une prophylaxie sanitaire efficace (5), mais elle est économiquement discutable sur des effectifs des bovins importés. Écarter de la reproduction les géniteurs reconnus comme transmettant le caractère "sensibilité à la dermatophilose » est, à coup sûr, plus logique.

\section{ESSAIS DE PROPHYLAXIE MÉdICALE}

Trois méthodes de prophylaxie médicale contre l'enzootie dermatophilosique ont été simultanément ou successivement tentées.

1) Douchage ou bain par des produits antibactériens.

2) Vaccination par voie intra-dermique.

3) Chimio-prévention par des injections d'antibiotique.

\section{Douchage ou bains par des produits anti- bactériens}

Cette méthode, très anciennement connue, garde toujours une grande valeur pratique. Sans résoudre totalement le problème de la dermatophilose (en particulier chez les races très sensibles), elle évite les grandes hécatombes. C'est après l'interruption forcée des douchages réguliers à la solution bovine " COOPER 》 que G. BUCK (2) déplore, en 1942,58 p. 100 de mortalité sur 600 bovins limousins croisés, et que nous avons nousmême noté 30 p. 100 des mortalités dans un troupeau de 103 Brahmans (10). Nombre de produits ont été recommandés par différents auteurs $(1,4,7)$, dont nous avons utilisé les quatre principaux : 
- ammoniums quaternaires, à la dilution 1/1000;

- solution bovine "COOPER 》 $\left(\mathrm{A}_{\mathrm{52}} \mathrm{O}_{3}\right.$ + crésyls + savon) à la dilution $1 / 250$;

- crésyls + savon, à la dilution $1 / 200$;

- sulfate de cuivre ou de zinc, à la dilution $1 / 400$.

A l'usage, ces produits se sont révélés d'intérêt à peu près équivalent, à condition d'être employés dans de bonnes conditions: produits frais, dilution optimale, application correcte sur tout le corps et emploi hebdomadaire en saison des pluies.

Comme pour les acaricides, il nous a paru intéressant d'alterner les produits lorsque leur efficacité semblait amoindrie (*).

L'emploi systématique des bains et douchages ne suffit pas à arrêter le développement de la dermatophilose, car il n'est pas toujours parfaitement applicable et les produits sont souvent lessivés par les pluies.

Aussi n'avons-nous pas quantifié avec précision le degré de protection qu'il confère contre la dermatophilose. Mais plusieurs expériences fortuites (rupture de stock du produit, panne des pompes de douchage, négligence du traitement de certains troupeaux) ont amplement prouvé que tout arrêt du douchage ou bain en saison de pluies entraîne une recrudescence immédiate de la maladie.

(*) Cette efficacité peut être utilement déterminée par un titrage in vitro. Nous avons réalisé ce titrage en mettant au contact des dilutions logarithmiques de la solution mère (stérilisée sur filtre Seitz) avec une culture en bouillon de Dermatophilus congolensis, dont nous avons ensuite apprécié la survie.

\section{Injection intradermique d'une culture vivante de Dermatophilus congolensis}

Au cours des années 1973 et 1974, nous avons tenté une vaccination contre la dermatophilose selon la méthode décrite par G. CHAMOISEAU et collab. (3) avec un vaccin élaboré et administré selon les indications des auteurs (*). $\mathrm{Au}$ cours de ces deux années, nous avons vacciné 156 zébus Brahmans ou croisés, au «Dermo-Jet » un mois avant la saison des pluies. Nous avons ensuite examiné mensuellement les lésions cutanées éventuelles sur chaque individu en même temps que sur sujets témoins, tous immatriculés au fer. Afin de ne comparer entre eux que des sujets de caractéristiques identiques (sexe, âge, état physiologique), nous avons dû réduire nos observations à 84 animaux vaccinés et 76 témoins.

Ces résultats sont résumés dans le tableau II.

On constate que la protection conférée est faible. Tout au plus peut-elle freiner l'impact de la maladie et éviter les épizooties trop sévères. La sensibilité extrême de la race Brahman explique peut-être cette difficulté d'immunisation par rapport aux zébus africains (3).

\section{Essais de chimio-prophylaxie : injection préventive de Spiramycine}

Par analogie avec les méthodes chimio-prophylactiques utilisées dans d'autres affections chroniques (maladies à protozoaires, tuberculose, etc...) nous avons songé à essayer la

(*) $\mathrm{La}$ dose vaccınale est de $0,2 \mathrm{ml}$ d'un mélange de deux culots de culture de 48 heures des souches B6M et B13M. L'injection de $1 \mathrm{ml}$ de ce mélange (avant centrifugation) à 40 lapins réduit de 20 à $30 \mathrm{p}$. 100 l'effet des scarifications expérimentales appréciées au $7^{\circ}$ jour.

TABL. N ${ }^{\circ}$ II-Résuittats des vaccinations (1973)

\begin{tabular}{|c|c|c|c|}
\hline \multicolumn{2}{|c|}{ Groupe des bovans vaccinés } & \multicolumn{2}{|c|}{ Graupe des bovins tëmoins } \\
\hline $\begin{array}{l}\text { Sujets en } \\
\text { expérience }\end{array}$ & $\begin{array}{l}\text { Sujets attengts de } \\
\text { dermatophilose }\end{array}$ & $\begin{array}{l}\text { Sujets en } \\
\text { expérience }\end{array}$ & $\begin{array}{l}\text { Sujets atteints de } \\
\text { dermatophilose }\end{array}$ \\
\hline 84 & $\begin{array}{l}\text { (45 à } 120 \text { jours après) } \\
32 \\
19 \text { atteunts légèrement } \\
6 \text { atteints gravement } \\
7 \text { atteints très gravement }\end{array}$ & 76 & $\begin{array}{l}\text { (45 à } 120 \text { jours après) } \\
41 \\
26 \text { atteints légèrement } \\
9 \text { atteints gravement } \\
6 \text { atteints très gravement }\end{array}$ \\
\hline $\begin{array}{l}\text { Soit un taux de } \\
(27 \text { à } 47 \mathrm{p} .100\end{array}$ & $\begin{array}{l}\text { dité de } 38,99 \text { p. } 100 \\
\text { sque } 5 \text { p. } 100 \text { ) }\end{array}$ & $\begin{array}{l}\text { Soit un taux } \\
(42 \text { a } 63 \mathrm{p} .10\end{array}$ & $\begin{array}{l}\text { idité de } 53,94 \text { p. } 100 \\
\text { sque } 5 \text { p. } 100 \text { ) }\end{array}$ \\
\hline
\end{tabular}


TABL. $N^{\circ}$ III-Résultats des traitements prëventifs à la spiramycine.

\begin{tabular}{|c|c|c|c|}
\hline \multicolumn{2}{|c|}{$\begin{array}{l}\text { Groupe des bovins pré-traitề à } \\
\text { la spiramycine }(25 \mathrm{mg} / \mathrm{kg})\end{array}$} & \multicolumn{2}{|c|}{ Groupe de bovins tềmoins } \\
\hline $\begin{array}{l}\text { Sujets en } \\
\text { expërience }\end{array}$ & $\begin{array}{l}\text { Sujets atteints de } \\
\text { dermatophilose }\end{array}$ & $\begin{array}{l}\text { Sujets en } \\
\text { expërience }\end{array}$ & $\begin{array}{l}\text { Sujets atteints de } \\
\text { dermatophilose }\end{array}$ \\
\hline 82 & $\begin{array}{l}\text { (30 à I20 jours après) } \\
7 \\
6 \text { atteints légèrement } \\
1 \text { atteint très gravement } \\
\text { (2 au } 45 \text { e jour, } 3 \text { au 60e jour } \\
\text { et } 2 \text { au } 90 \mathrm{e} \text { jour })\end{array}$ & 81 & $\begin{array}{l}\text { (30 à } 120 \text { jours après) } \\
19 \\
14 \text { atteints légèrement } \\
4 \text { atteints gravement } \\
1 \text { atteint très gravement }\end{array}$ \\
\hline \multicolumn{2}{|c|}{$\begin{array}{l}\text { Soit un taux de morbidité de } 8,53 \mathrm{p} .100 \\
\text { (3 à } 16 \mathrm{p} .100 \text {, au risque } 5 \text { p. 100) }\end{array}$} & \multicolumn{2}{|c|}{$\begin{array}{l}\text { Sojt un taluxde morbidite de } 23,45 \mathrm{p}, 100 \\
(15 \text { à } 35 \mathrm{p} .100 \text {, au risque } 5 \mathrm{p} .100)\end{array}$} \\
\hline
\end{tabular}

valeur d'une chimio-prévention de la dermatophilose. A l'occasion de nombreux essais préliminaires sur lapins (10), nous avons éliminé plusieurs produits (Lipiodol, Crésyl, Trypanocides, etc...) pour ne conserver qu'un antibiotique à pouvoir rémanent et fixation tissulaire : la Spiramycine $(*)$.

Cet antibiotique a donc été injecté mensuellement à 103 bovins, à la dose de $25 \mathrm{mg} / \mathrm{kg}$, par voie musculaire. Les lésions cutanées de chaque sujet, ainsi que celles de témoins correspondants, ont été appréciées durant les 4 mois suivant l'injection. Seuls 82 sujets, pour lesquels nous disposions de témoins identiques, ont pu être utilisés.

Les résultats sont résumés dans le tableau III.

(*) Suanovil (Specia) solution à 20 p. 100 . Injecté par voie intramusculaire à 40 lapins $(25 \mathrm{mg} / \mathrm{kg}$ ) cet antibiotique réduit de 23 p. 100 l'effet des scarifications expérimentales au $7^{\mathrm{e}}$ jour, même 15 jours après l'injection.
On constatera que, comme dans le cas de la vaccination, la proportion de sujets protégés est faible. Elle est loin d'entraîner une sécurité valable contre la maladie, et reste d'un prix de revient élevé.

\section{CONCLUSION}

L'arme prophylactique idéale contre la dermatophilose reste encore à découvrir. Compte tenu de l'étio-pathogénie de la maladie en élevage extensif elle sera probablement médicale.

Un progrès certain peut être apporté par une chimio-prophylaxie mensuelle (par voie externe ou parentérale) ou par la vaccination annuelle, moins onéreuse et plus pratique.

Néanmoins, les protections conférées par chacune de ces méthodes ne sont pas toujours cumulables et la dermatophilose des zébus Brahmans, si elle est en partie maîtrisable, est loin d'être vaincue à Madagascar.

\section{SUMMARY}

\section{Control of dermatophilus congolensis infection}

in Brahman cattle. Results after seven years

Results of sanitary and medical prophylaxis are compared.

Medical prophylaxis is likely more efficient, either as a chimio-prophylaxıs (diping or preventive injections of antibiotics) or as a vaccination. But it cannot always control the disease in Brahman cattle.

\section{RESUMEN}

Balance de siete años de profilaxia de la dermatofilosis en una manada de cebues Brahman

Se comparan los resultados de las profilaxias sanitaria y medical. La última parece más eficaz, sea bajo forma de una quimioprofilaxia mensual (por via externa o parenteral) sea de una vacunación anual.

Pero no es suficiente para proteger la totalidad de una manada contra la enfermedad en ganaderia extensiva. 


\section{BIBLIOGRAPHIE}

1. BLANCOU (J.). The treatment of infection by Dermatophilus congolensis with particular reference to the disease in cattle. Int. Symp. on Dermatophilus Infection University of Ibadan (Nigeria), 25, 28 iuin 1973. New-York, Academic Press, 1975.

2. BUCK (G.). Actinomycose ou streptothricose cutanée des bovins (Drodro, Boka). Bull. Off. int. Epiz., 1948, 29 (3-4) : 117-121.

3. CHAMOISEAU (G.), PROVOST (A.), TOUADE (M.). Recherches immunologiques sur la dermatophilose cutanée bovine. II. - Essais d'ımmunisation du bœuf contre la dermatophilose naturelle. Rev. Elev. Méd. vét. Pays trop., 1973, 26 (1) 7-4.

4. CURASSON (G.). Traité de pathologie exotique vétérinaire et comparée. Tome II. Paris, Vigot, 1942.

5. DUMAS (R.), LHOSTE (P.), CHABEUF (N.), BLANCOU (J.). Note sur la sensibilité héréditaire des bovins à la streptothricose. Rev. Elev. Méd. vét. Pays trop., 1971, 24 (3): 349-353.

6. GRABER (M.). Existence au Tchad des taurins et de zébus porteurs sains de Dermatophilus congolensis. Rev. Elev. Méd. vét. Pays trop., 1969, 22 (1): 41-45.

7. LE RICHE (P. D.). The activity of dipping fluids in treatment and prevention of mycotic dermatitis in sheep. Aust. vet. J., 1967, 43 (7) : 265-269.

8. MACADAM (I.). Some observations on bovine cutaneous streptothricosis in Northern Nigeria. Trop. anim. Hith. Prod., 1970, (2) : 131-138.

9. PERREAU (P.). Maladies tropicales du bétall. Parıs, P. U. F., 1973. (Coll. techn. vivantes.)

10. Rapports annuels du Laboratoire Central de l'Elevage, Tananarive. Arch. I. E. M. V. T. (MaisonsAlfort) et M. D. R. (Tananarıve). 